\title{
Mapping and characterization of novel parthenocarpy QTLs in tomato
}

\author{
Benoit Gorguet $\cdot$ Pieter Martijn Eggink · Juan Ocaña • \\ Aparna Tiwari - Danny Schipper · Richard Finkers · \\ Richard G. F. Visser · Adriaan W. van Heusden
}

Received: 9 October 2007 / Accepted: 20 December 2007/Published online: 30 January 2008

(C) The Author(s) 2008

\begin{abstract}
Parthenocarpy is the development of the fruit in absence of pollination and/or fertilization. In tomato, parthenocarpy is considered as an attractive trait to solve the problems of fruit setting under unfavorable conditions. We studied the genetics of parthenocarpy in two different lines, IL5-1 and IVT-line 1, both carrying Solanum habrochaites chromosome segments. Parthenocarpy in IL5-1 is under the control of two QTLs, one on chromosome 4 (pat4.1) and one on chromosome 5 (pat5.1). IVT-line 1 also contains two parthenocarpy QTLs, one on chromosome 4 (pat4.2) and one on chromosome 9 (pat9.1). In addition, we identified one stigma exsertion locus in IL5-1, located on the long arm of chromosome 5 (se5.1). It is likely that pat4.1, from IL5-1 and pat4.2, from IVT-line 1, both located near the centromere of chromosome 4 are allelic. By making use of the microsynteny between tomato and Arabidopsis in this genetic region, we identified $A R F 8$ as a potential candidate gene for these two QTLs. ARF8 is known to act as an inhibitor for further carpel development in Arabidopsis, in absence of pollination/fertilization. Expression of an aberrant form of the Arabidopsis ARF8 gene, in tomato, has been found to cause parthenocarpy. This candidate gene approach may lead to the first isolation of a
\end{abstract}

Communicated by I. Paran.

B. Gorguet · P. M. Eggink · J. Ocaña · A. Tiwari ·

D. Schipper · R. Finkers - R. G. F. Visser .

A. W. van Heusden ( $\square)$

Graduate School of Experimental Plant Sciences,

Wageningen UR Plant Breeding, PO Box 386,

6700AJ Wageningen, The Netherlands

e-mail: sjaak.vanheusden@wur.nl

B. Gorguet

e-mail: benoit.gorguet@wur.nl parthenocarpy gene in tomato and will allow further use in several crop species.

\section{Introduction}

In normal fruit development, the initiation of fruit set depends on the successful completion of pollination and fertilization. However, these processes depend on narrow environmental constrains (Picken 1984). Normal pollen production is restricted to a specific temperature range, and mechanical vibration of the flowers, either manually or by bees is necessary to ensure pollen shedding. In tomato, failure to set fruit is therefore a common phenomenon under certain field conditions (high humidity combined with low or high temperatures) and in unheated greenhouses or tunnels during Winter or early Spring cultivation (George et al. 1984). Parthenocarpic fruit development, which is the growth of the ovary into a seedless fruit in the absence of pollination and/or fertilization, offers an opportunity to overcome this problem of poor fruit set under unfavorable conditions. In tomato three sources (distinct varieties) of natural parthenocarpy have been widely studied because of their perspectives for practical application to produce seedless fruits (reviewed by Gorguet et al. 2005): Soressi or Montfavet 191 (pat), Severianin (pat-2) and RP75/59 (pat-3/pat-4). In addition, two other sources of parthenocarpy in tomato, IVT-line 1 and 2 (Zijlstra 1985) were found to give a higher and more stable level of parthenocarpy than Soressi and Severianin. IVTline 1 (Fig. 1) was developed in the early 1980s from an interspecific cross between Solanum habrochaites (accession unknown) and S. lycopersicum, followed by several generations of backcrosses and by at least one selfing 


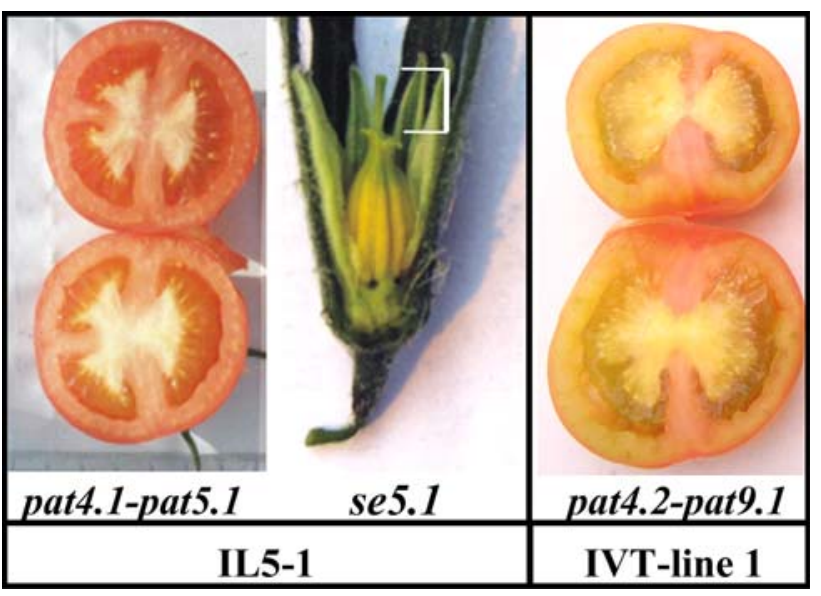

Fig. 1 Seedless fruits observed on IL5-1 (left picture) and IVT-line 1 (right picture). Stigma exsertion observed on IL5-1 (middle picture)

(Zijlstra 1985). This line was developed specifically for parthenocarpy and it was thought to be controlled by one single gene. Parthenocarpy in IVT-line 2 originated from $S$. peruvianum and was assumed to be polygenic (Zijlstra 1985). To date, the only mapped gene for parthenocarpy in tomato is pat, localized on the long arm of chromosome 3 (Beraldi et al. 2004). However, even in conditions favorable for seed production, pat genotypes give a very low seed set (Mazzucato et al. 1998), which makes it less attractive for practical breeding.

Recently Finkers et al. (2007b) have developed a set of introgression lines (ILs) for $S$. habrochaites accession LYC4 in the S. lycopersicum cv. Moneymaker genetic background. Parthenocarpic fruit development and stigma exsertion were observed in one of the ILs (IL5-1; R. Finkers, personal communication).

In this study we characterized and mapped four novel parthenocarpy QTLs responsible for the seedless fruit development in IL5-1 and IVT-line 1. In addition the position of the stigma exsertion locus, in IL5-1, was also identified. The syntenic relationship between two parthenocarpy QTLs and the ARF8 Arabidopsis parthenocarpy gene (Goetz et al. 2006) was investigated. ARF8 was determined as a likely candidate for these two parthenocarpy QTLs.

\section{Materials and methods}

Plant materials

For the mapping procedure in S. habrochaites LYC4, we originally used two $\mathrm{BC}_{5} \mathrm{~S}_{1}$ populations that were part of the IL development program of Finkers et al. (2007b). These ILs contain chromosome fragment(s) of S. habrochaites LYC4, hereafter referred to as $S H$, in a $S$. lycopersicum cv. Moneymaker genetic background, hereafter referred to as $S L$. IL5-1 carries an $S H$ introgression on the short arm of chromosome 4 and the complete chromosome 5 from SH. IL5-2 carries the long arm of chromosome 5 from $\mathrm{SH}$. The two $\mathrm{BC}_{5} \mathrm{~S}_{1}$ populations had previously been used for the selection of IL5-1 and IL5-2 (Finkers et al. 2007b) and will be hereafter denoted as populations 5-1 and 5-2. Population 5-1, segregating for the short arm of chromosome 4 and the entire chromosome 5, consisted of 174 plants. Population 5-2, segregating for the long arm of chromosome 5, was composed of 183 plants. Plants were grown in Summer/ Autumn 2005 in a heated glasshouse in Wageningen, The Netherlands, under controlled conditions (temperature always superior to $15^{\circ} \mathrm{C}$ ). The average length of days during fruit set was $\sim 12 \mathrm{~h}$. Subsequently a set of relevant $\mathrm{BC}_{5} \mathrm{~S}_{2}$ recombinant plants were selected and further studied in Spring/Summer 2006, with an average length of days during fruit set of $\sim 14 \mathrm{~h}$, and in Winter 2006/2007, with an average length of days during fruit set of $\sim 9 \mathrm{~h}$ and lower light intensity than in Summer.

For the mapping of parthenocarpy genes in IVT-line 1, we used an $F_{2}$ population coming from a single cross between the parthenocarpic IVT-line 1, and the nonparthenocarpic $S$. lycopersicum cv. Moneymaker. The $\mathrm{F}_{2}$ population of this cross was composed of 160 plants and grown under controlled conditions, as above, in Spring 2006, with an average length of days during fruit set of $\sim 13 \mathrm{~h}$.

\section{Flower morphology}

Fresh flowers were collected and analyzed at pre-anthesis on the third flower truss of $\mathrm{BC}_{5} \mathrm{~S}_{1}$ plants. In this experiment pre-anthesis was defined as the flower stage in which the sepals start opening, and the color of the anther cone start turning from green to yellow. Style length, ovary length and stamen length were measured to the nearest $0.1 \mathrm{~mm}$. Stigma exsertion was determined by subtracting stamen length from the sum of the style length plus the ovary length, as presented by Chen and Tanksley (2004).

\section{Characterization of parthenocarpy}

In facultative parthenocarpy, the development of seedless fruits can only be observed on the flowers that are not pollinated. To minimize pollination, flowers were not vibrated. To classify the level of parthenocarpy, the first five fruit clusters of a plant were analyzed for fruit size, number of fruits, number of flowers and the presence of 
seeds. The diameter of the fruits was measured to the nearest $1 \mathrm{~mm}$, using a slide caliper. Fruits were scored at mature stage. The trait parthenocarpy was calculated quantitatively, as the percentage of parthenocarpic fruits from the total number of flowers over the first five clusters. This percentage is hereafter referred to as "parthenocarpy level". To be considered parthenocarpic, the fruits had to be fully seedless, of the same shape than a seeded fruit (round and not angular), of the same color aspect (shiny and not pale), and have jelly filled locules.

\section{DNA extraction}

Two DNA isolation techniques were used. For most experiments total DNA was isolated from two young tomato leaves by using a CTAB DNA isolation method as described by Steward and Via (1993), adjusted for 96-well format using $1.2 \mathrm{ml}$ COSTAR cluster tubes (Corning Incorporated). Leaf samples were crushed using a Retsch $300 \mathrm{~mm}$ shaker at $30 \mathrm{r} / \mathrm{s}$ for $45 \mathrm{~s}$ (Retsch BV).

DNA isolation, for the selection of relevant $\mathrm{BC}_{5} \mathrm{~S}_{2}$ progenies, was performed by a rapid alkaline $(\mathrm{NaOH})$ based extraction method (Wang et al. 1993). This method was up-scaled to a 96-well format as described by Gorguet et al. (2006).

\section{Molecular marker analysis}

Genotypes were determined using PCR-based markers. Primers and enzymes of CAPS and SCAR markers TG441, CD64, CD31, TACL2, TG538, TG318 have been described by Coaker and Francis (2004) and TG358 by Brouwer and St. Clair (2004). Other CAPS and SCAR markers were generated based on RFLP and COS marker sequences previously mapped by Tanksley et al. (1992) or Fulton et al. (2002). The sequences of the RFLP and COS markers were available on the "SOL Genomics Network" (Mueller et al. 2005; http://sgn.cornell.edu). The conversion of RFLP and COS markers into CAPS and SCAR markers was performed as described in Gorguet et al. (2006). Details on these markers are given in Table 1. Each PCR reaction $(25 \mu \mathrm{l})$ contained $\sim 20 \mathrm{ng}$ of genomic DNA, $1 \times$ PCR-reaction buffer, $0.4 \mu \mathrm{M}$ of each forward and reverse primer, $0.2 \mathrm{mM}$ dNTPs and $0.5 \mathrm{U}$ Taq polymerase in miliQ water. PCR conditions were: hot start of $5 \mathrm{~min}$ at $94^{\circ} \mathrm{C}$, followed by 39 cycles of $30 \mathrm{~s}$ at $94^{\circ} \mathrm{C}, 30 \mathrm{~s}$ at annealing temperature (Table 1), $30 \mathrm{~s}$ at $72^{\circ} \mathrm{C}$ and a final extension of $7 \mathrm{~min}$ at $72^{\circ} \mathrm{C}$. About $3 \mu$ of PCR product was digested in a total volume of $15 \mu \mathrm{l}$ for at least $3 \mathrm{~h}$ with $2 \mathrm{U}$ of restriction enzyme. After digestion, DNA fragments were separated on a $2 \%$ agarose gel. Reverse primers for microsatellite markers were labeled with IRD700 or IRD800. PCR reactions $(10 \mu \mathrm{l})$ were prepared in the same proportion as described for CAPS markers, only with $0.1 \mu \mathrm{M}$ forward and labeled reverse primer. PCR conditions were: hot start of $3 \mathrm{~min}$ at $94^{\circ} \mathrm{C}$, followed by 30 cycles of $45 \mathrm{~s}$ at $94^{\circ} \mathrm{C}, 45 \mathrm{~s}$ at $53^{\circ} \mathrm{C}, 1 \mathrm{~min}$ at $72^{\circ} \mathrm{C}$ and a final extension of $3 \mathrm{~min}$ at $72^{\circ} \mathrm{C}$. After the PCR, $10 \mu \mathrm{l} \mathrm{LI-COR} \mathrm{loading}$ dye was added and the IRD700 labeled fragments were analyzed on a LI-COR 4200 DNA sequencer, essentially following the method published by Myburg and Remington (2000).

AFLP markers were determined as described by Gorguet et al. (2006), using the same primer combinations as presented in Finkers et al. (2007a).

\section{Data analysis and mapping}

To normalize the distribution of the recorded trait, the parthenocarpy level (percentage of seedless fruits) was transformed to a logit scale: $\operatorname{logit}(p)=\log (p /(100-p))$, (with $p$ the percentage of seedless fruits on the first five clusters per plant).

Genetic linkage maps were constructed with JoinMap 3.0 (Van Ooijen and Voorrips 2001), applying the Kosambi mapping function. QTL mapping was performed using the interval mapping and multiple-QTL mapping procedures of MapQTL 5 (Van Ooijen 2004). A logarithm of odds (LOD) threshold value of 3.0 was set (Van Ooijen 1999). A twoLOD support interval was taken as a confidence interval for a putative QTL. Models for QTL analysis are presented hereafter.

In the $\mathrm{BC}_{5} \mathrm{~S}_{2}$ population, the linear model used for the phenotype $Y$ of an individual was:

$Y_{i(j)}=\mu+X_{i(j)} \alpha_{j}+e_{i} \quad[\operatorname{model} 1]$

where $\mu$ is the population mean, $X_{i(j)}$ is the number of $S H$ alleles at the major locus for individual $i(j)$ and $\alpha_{j}$ is the effect of one allele of the major QTL. This effect differs according to $j$, the genotypic status of the minor QTL. $j=1(i=1, \ldots, 61)$ when the minor QTL is homozygous $S L ; j=2(i=1, \ldots, 21)$ when the minor QTL is heterozygous and $j=3(i=1, \ldots, 24)$ when the minor QTL is homozygous $S H . e_{i}$ is the residual.

In the $F_{2}$ population used for the mapping of two parthenocarpy QTLs, the model used for the phenotype $Y$ of an individual was the factorial combination of the two loci:

$Y=\mu+X_{1} X_{2}+e \quad[$ model 2$]$

where $\mu$ is the population mean, $X_{1} X_{2}$ is the effect of the combinations of the two parthenocarpy QTLs and $e$ is the residual. 
Table 1 Primer sequences and PCR reaction parameters for CAPS and SCAR markers

\begin{tabular}{|c|c|c|c|c|c|c|c|}
\hline Name & Primer $\left(5^{\prime}-3^{\prime}\right)$ forward, reverse & $\operatorname{Size}^{\mathrm{a}}(\mathrm{bp})$ & $\mathrm{TA}^{\mathrm{b}}\left({ }^{\circ} \mathrm{C}\right)$ & $\mathrm{Chr}^{\mathrm{c}}$ & Restriction enzyme & $S L \operatorname{size}^{\mathrm{e}}$ & $S H$ size $^{\mathrm{e}}$ \\
\hline TG609 & $\begin{array}{l}\text { ATATGACTAGGAGGCAATGACTGA } \\
\text { TTGCCTACTTATAACCCTGTGGA }\end{array}$ & 400 & 52 & 4 & $A l u \mathrm{I}$ & 400 & 280 \\
\hline CT258 & $\begin{array}{l}\text { CAATGAATCATCTGTGGTGATT } \\
\text { TGCATTCCTCTGTGGATGCT }\end{array}$ & 200 & 55 & 4 & HinfI & 80 & 150 \\
\hline C2_At3g24010 & $\begin{array}{l}\text { ATGCAATCAGGATTGCTGATG } \\
\text { CTGATCGAGCTGCTGAATATG }\end{array}$ & 1,000 & 55 & 9 & $\operatorname{Taq} \mathrm{I}$ & 1,000 & 600 \\
\hline T0156 & $\begin{array}{l}\text { GCGGTTGATTCACATCGTAA } \\
\text { CCTGTAGCACCCAAAGGATG }\end{array}$ & 1,100 & 55 & 9 & НруСН4IV & 1,100 & 550 \\
\hline CT220 & $\begin{array}{l}\text { AAGCGAATTATCTGTCAAC } \\
\text { GTTCCTGACCATTACAAAAGTAC }\end{array}$ & 200 & 55 & 9 & MseI & 100 & 130 \\
\hline T1065 & $\begin{array}{l}\text { GACGGTGAAGGGTACCAAG } \\
\text { CAGGAGTGCATGGGTAGGT }\end{array}$ & 550 & 55 & 9 & $S s p \mathrm{I}$ & 550 & 400 \\
\hline C2_At5g06360 & $\begin{array}{l}\text { GGCTATGCATGAAGAGTCATC } \\
\text { GGCACCTCCCATTTTCCAGC }\end{array}$ & 250 & 55 & 9 & ApoI & 200 & 250 \\
\hline СТ229 & $\begin{array}{l}\text { ATGGGCTGGGATCGTAGTAAA } \\
\text { AAGCTTGCGATTCCCATAACAT }\end{array}$ & 336 & 55 & 4 & MwoI & 300 & 336 \\
\hline T0208 & $\begin{array}{l}\text { AACGCCCCAGCCTGACTACA } \\
\text { CTGGGGAGGTTTCGATTTCTG }\end{array}$ & 514 & 55 & 4 & HindIII & 514 & 480 \\
\hline TG483 & $\begin{array}{l}\text { CACTCCCATGGCAGATAAAA } \\
\text { AGTGAAGTAAAACAAAGCCAAAAT }\end{array}$ & 334 & 59 & 4 & $H p h \mathrm{I}$ & 334 & 200 \\
\hline Т0703 & $\begin{array}{l}\text { ATTTTTACGGGCAAGCGACTG } \\
\text { CGTTGATCCCTCTATAATGGTG }\end{array}$ & 456 & 55 & 4 & НруСН4IV & 350 & 250 \\
\hline T1068 & $\begin{array}{l}\text { CAAAGCAATGGGCAATGGT } \\
\text { ACACAGCAGTTTCAGTAGGAC }\end{array}$ & 500 & 55 & 4 & HincII & 400 & 500 \\
\hline CT175 & $\begin{array}{l}\text { CAGCTAAGCGTTGACAGTTGAGAA } \\
\text { ATGGCCGCGGTTTGAGC }\end{array}$ & 750 & 55 & 4 & MseI & 200 & 280 \\
\hline TG182 & $\begin{array}{l}\text { GCTCGGGCAACAGTGAAC } \\
\text { GCTAAGCAAATGAAAAACCAGA }\end{array}$ & 335 & 55 & 4 & $T a q \mathrm{I}$ & 335 & 280 \\
\hline TG370 & $\begin{array}{l}\text { ATGCTGCTGCCGGTTCCACT } \\
\text { ATCGGGTCTCTAATTTCAGCAC }\end{array}$ & 352 & 55 & 4 & НруСН4IV & 352 & 200 \\
\hline T0958 & $\begin{array}{l}\text { GTGTCGAACCCTTGGCAACAAT } \\
\text { AGTTCTTTCAGCTTTTGGGTTAA }\end{array}$ & 650 & 55 & 4 & $R s a \mathrm{I}$ & 400 & 300 \\
\hline T0891 & $\begin{array}{l}\text { GACCGCTACCTCAACTTCT } \\
\text { CACTCTAATACTCCACTCAACATA }\end{array}$ & 1,200 & 55 & 4 & DraI & 1,200 & 700 \\
\hline TG339 & $\begin{array}{l}\text { GAAACCTTACCCCTCTA } \\
\text { CGCTGTTTCTTGCCATTT }\end{array}$ & $\begin{array}{l}436 \\
500^{\mathrm{d}}\end{array}$ & 46 & 4 & & & \\
\hline T0529 & $\begin{array}{l}\text { TGGAGAGGAACAGGCTAAATC } \\
\text { CACTCCGGCAACTGAAATGT }\end{array}$ & $\begin{array}{l}1,650 \\
1,600^{\mathrm{d}}\end{array}$ & 55 & 4 & & & \\
\hline T0635 & $\begin{array}{l}\text { CCAGAACCTCGACTCATCA } \\
\text { TAGCCTCACAGTCTCAGTCAA }\end{array}$ & 300 & 55 & 4 & HincII & 80 & 100 \\
\hline TG60 & $\begin{array}{l}\text { TTGGCTGAAGTGAAGAAAAGTA } \\
\text { AAGGGCATTGTAATATCTGTCC }\end{array}$ & 1500 & 55 & 5 & НруСН4IV & 350 & 520 \\
\hline CT138 & $\begin{array}{l}\text { ACCAGCCCCGGAAGATTTTA } \\
\text { GCGGTCAACTTCAGCAACTAT }\end{array}$ & 900 & 55 & 5 & $R s a \mathrm{I}$ & 700 & 600 \\
\hline
\end{tabular}

\footnotetext{
a Size of undigested PCR product

b PCR annealing temperature

c Chromosome number

${ }^{\mathrm{d}}$ PCR product size on Solanum lycopersicum and S. habrochaites, respectively

e Band size estimation after digestion, on S. lycopersicum $(S L)$ and S. habrochaites $(S H)$
} 


\section{Results}

Parthenocarpic fruit development was observed in the introgression line IL5-1 developed by Finkers et al. (2007b; Fig. 1), but not characterized into details. In addition, flowers of IL5-1 presented an exserted stigma from pre-anthesis stage on. In such a phenotype, selfpollination is altered because the stigma is out of the anther cone when the pollen is released inside the anther cone (Fig. 1). We hypothesized that the observed seedless fruits setting in IL5-1 was due to a combination of parthenocarpy and functional sterility. Functional sterility is characterized by a normal development of viable pollen, but natural pollination is strongly restricted due to some deviation from the normal morphology and function of the flower. Because ILs were initially vibrated to promote pollination, parthenocarpic fruit development was only obvious with the presence of a certain form of sterility. IL5-2, carrying only the long arm of chromosome 5 of $\mathrm{SH}$, was not parthenocarpic but showed stigma exsertion. We concluded that the stigma exsertion locus was located on chromosome 5 .

To map and characterize the parthenocarpy and functional sterility traits observed in this material, we generated a genetic linkage map of the introgressed regions of the two ILs by making use of two $\mathrm{BC}_{5} \mathrm{~S}_{1}$ populations: population 51 and population 5-2 segregating for the $S H$ introgressions of IL5-1 and IL5-2, respectively. The recurrent parent used for the development of IL5-1 and IL5-1, cv. Moneymaker $(S L)$, does not produce any parthenocarpic fruit, under the criteria presented in the "Materials and methods". Therefore the parthenocarpy loci of IL5-1 are located in the $\mathrm{SH}$ introgressions.

Because the expression of parthenocarpy requires that the plants are not pollinated, we selected them for functional sterility. For this, both populations were screened at juvenile stage with SCAR marker TG318, to select for plants that are homozygous or heterozygous $\mathrm{SH}$ at that locus. The final population 5-1 and population 5-2 consisted of 74 and 66 plants, respectively. Hereafter, "populations 5-1 and 5-2" refer to these selected plants. TG318 was chosen to screen the populations because it is located in the middle of the chromosome 5, near the centromere, and it carries the alleles of $\mathrm{SH}$ in both IL5-1 and IL5-2. Therefore TG318 was likely to be near the stigma exsertion locus. By skipping the plants homozygous $S L$ at TG318 locus in the segregating populations, we enriched them for plants with functional sterility, to promote visible parthenocarpic fruit development. Subsequently, parthenocarpy was evaluated in population 5-1 and functional sterility in populations 5-1 and 5-2.
Genetic linkage map construction

The initial step in the development of linkage maps in the regions of the $S H$ introgressions was to identify markers on the borders of the introgression. We developed a set of RFLP- and COS-derived PCR primer combinations in the expected regions of the introgressions and determined whether the loci were in or out. The border of the introgression on chromosome 4 in IL5-1 was determined between markers T0635 and TG609, respectively, at 55 and $56 \mathrm{cM}$ on the EXPEN2000 linkage map (Fulton et al. 2002). On chromosome 5 , the border of the introgression in IL5-2 was identified between markers CD64 and CD31, respectively, at 27 and $39 \mathrm{cM}$ on the EXPEN1992 linkage map (Tanksley et al. 1992). SH introgression of IL5-1 is likely to cover the entire chromosome 5 (Finkers et al. 2007b).

The genetic linkage map of the $S H$ chromosome 4 introgression of IL5-1 was generated using the population 5-1 $(n=74)$. Thirteen RFLP or COS markers located on the short arm of chromosome 4 in the EXPEN2000 map were converted into CAPS or SCAR markers and mapped in population 5-1. Two microsatellite markers, SSR43 and SSR72 (http://sgn.cornell.edu; Mueller et al. 2005), were added. The introgression on chromosome 4 spanned $22.4 \mathrm{cM}$, which is almost the complete short arm of chromosome 4, from the telomere to CAPS marker T0635 (Fig. 3a).

The genetic linkage map of the $S H$ chromosome 5 was constructed using populations 5-1 and 5-2 separately. A total of nine CAPS or SCAR markers were developed either based on available information (Brouwer and St. Clair 2004; Coaker and Francis 2004) or based on RFLP sequences (Tanksley et al. 1992). The $S H$ introgression of IL5-2 spanned 21.1 cM on the long arm of chromosome 5, from the telomeric end to CAPS marker CD31. The limit of the $\mathrm{SH}$ introgression of IL5-1 on the short arm of chromosome 5 (distal to TG441), was not determined, therefore the introgression spanned at least $77.2 \mathrm{cM}$ (Fig. 3a). Because populations 5-1 and 5-2 were selected for homozygous and heterozygous $S H$ alleles at TG318 locus, the recombination ratio was underestimated around the TG318 locus, which reduced the distances in the chromosome 5 introgressions of IL5-1 and IL5-2. However, this did not affect the order of the markers and therefore did not affect the relative positions of the subsequent mapped loci.

The order of the markers on chromosomes 4 and 5 were in accordance with the Tomato-EXPEN2000 map and EXPEN1992 map of the "SOL Genomics Network" (http://sgn.cornell.edu). Overall the map distance in the chromosome 4 introgression was reduced to $41 \%$ of the distance found in the same interval in the EXPEN2000 
reference map. Regarding the chromosome 5, the map distance was quasi equal $(92 \%)$ between IL5-1 and the EXPEN1992 reference map (Tanksley et al. 1992). In contrast, the map distance in the chromosome 5 introgression of IL5-2 was reduced to $38 \%$ of the distance observed in the EXPEN1992 reference map.

\section{Screening and mapping of parthenocarpy}

The distribution of the parthenocarpy level in population 51 ranged from 0 to $90.5 \%$ (Fig. 2) and the average size of the parthenocarpic fruits $(4.73 \mathrm{~cm})$ did not significantly differ $(P>0.05)$ from the size of the seeded fruits $(4.75 \mathrm{~cm})$.

By applying interval mapping, one QTL for parthenocarpy (designated pat4.1) was identified on chromosome 4 (Fig. 3a), close to the centromere, with the highest LOD value at CAPS markers T0958/T0891/T0635 (Table 2). This QTL explained $48.9 \%$ of the total variation. By using one of the three peak markers as cofactor, in an MultipleQTL Mapping (MQM) procedure, no extra QTL was detected in the introgressions.

\section{Confirmation of parthenocarpy QTLs in $\mathrm{BC}_{5} \mathrm{~S}_{2}$}

In order to confirm and narrow down the confidence interval of pat4.1 on chromosome 4 and to study the potential interaction of pat4.1 with genes on chromosome 5 , we developed a set of recombinant progenies with small

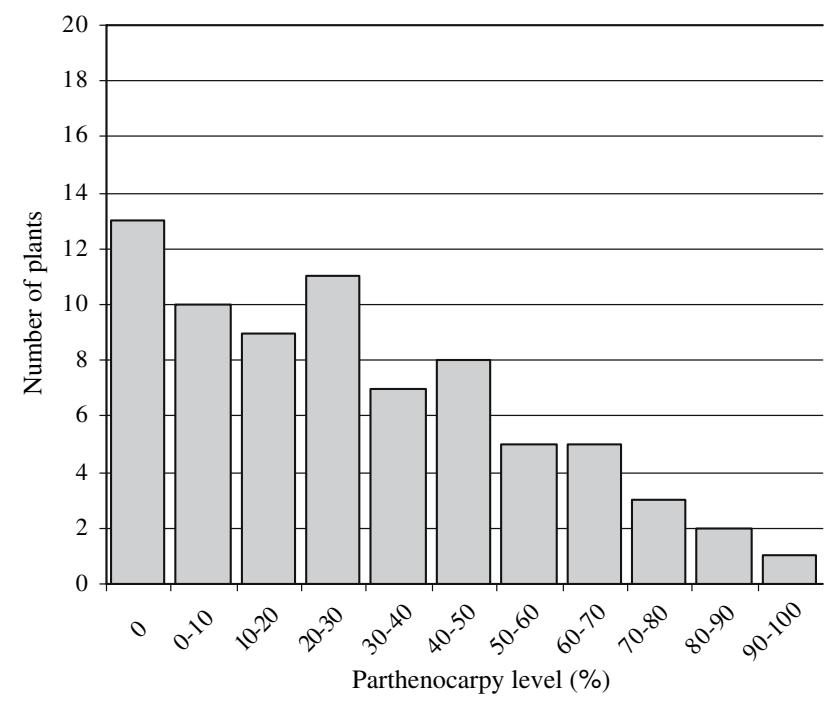

Fig. 2 Frequency distribution of the parthenocarpy levels (percentages) over the first five clusters in the $\mathrm{BC}_{5} \mathrm{~S}_{1}$ population 5-1 $(n=74)$. i.e. " 0 " refers to plants without seedless fruit; " $0-10$ " refers to plants with at least one seedless fruit and less than $10 \%$ of the flowers setting seedless fruits homozygous $S H$ introgressions on the short arm of chromosome 4. To develop this set of progenies, $\mathrm{BC}_{5} \mathrm{~S}_{2}$ plants, recombinant for the chromosome 4 introgression, were screened at seedling stage using molecular markers. Homozygous recombinant plants were selected and divided into nine classes (Fig. 4). Dominant parthenocarpy QTLs on chromosome 5 could not have been identified in the previous population $\left(\mathrm{BC}_{5} \mathrm{~S}_{1}\right)$ because most plants were homozygous $\mathrm{SH}$ or heterozygous for chromosome 5 due to the enrichment for plants with functional sterility. The evaluation of the recombinant progenies segregating for chromosome 5 allowed us to look for potential dominant parthenocarpy QTLs on chromosome 5. Selected plants were genotyped and their parthenocarpy level was assessed from April to July 2006 (hereafter referred to as Spring/ Summer). After making cuttings, the recombinant progenies were grown and re-evaluated in Winter 2006/2007 (hereafter referred to as Winter). The parthenocarpy level of each recombinant progeny is presented in Fig. 4. Only chromosome 4 recombinant progenies carrying the $\mathrm{SH}$ chromosome segment TG182-T0635 produced parthenocarpic fruits. This narrows down the position of the parthenocarpy QTL pat4.1 confidence interval to $3.7 \mathrm{cM}$ in population 5-1. The parthenocarpy level was significantly higher in Spring/Summer than in Winter $(P<0.05)$. More strikingly however, was the great variation of parthenocarpy level, from one progeny to another. This did not depend on the size of the $S H$ fragment on chromosome 4, eliminating the possibility of having a second parthenocarpy QTL on that chromosome. Therefore we investigated the potential interaction of pat4.1 with chromosome 5 in progenies 1-5. To study this interaction we applied the Multiple-QTL mapping (MQM) function and used marker T0635 (one of the peak markers for pat4.1) as co-factor. A QTL linked to marker CD64 on chromosome 5 showed a significant effect on the expression of the parthenocarpy trait (Fig. 3a). This QTL is hereafter referred to as pat5.1 and was detected in Spring/Summer and Winter.

In order to study the effects and interaction of pat4.1 and pat5.1 (Table 3), we searched for the best linear model to explain the observed variation. pat4.1 is clearly the main QTL in this interaction, but the size of its effect depends on the alleles of pat5.1. A higher level of parthenocarpy is observed when the two $S H$ alleles of pat4.1 are present in combination with at least one $S H$ allele of pat5.1. In Spring/Summer, the parthenocarpy level observed on these plants is on average $41 \%$ with one $S H$ allele of pat5.1 and $46 \%$ with two $\mathrm{SH}$ alleles (no significant difference). It is not clear whether pat4.1 in heterozygous, in combination with pat5.1 homozygous $S H$ gives also a high level of parthenocarpy, because only one plant had this specific genotype. When pat4.1 is homozygous $S L$, the pat5.1 QTL alone never shows parthenocarpy. Regarding these two 


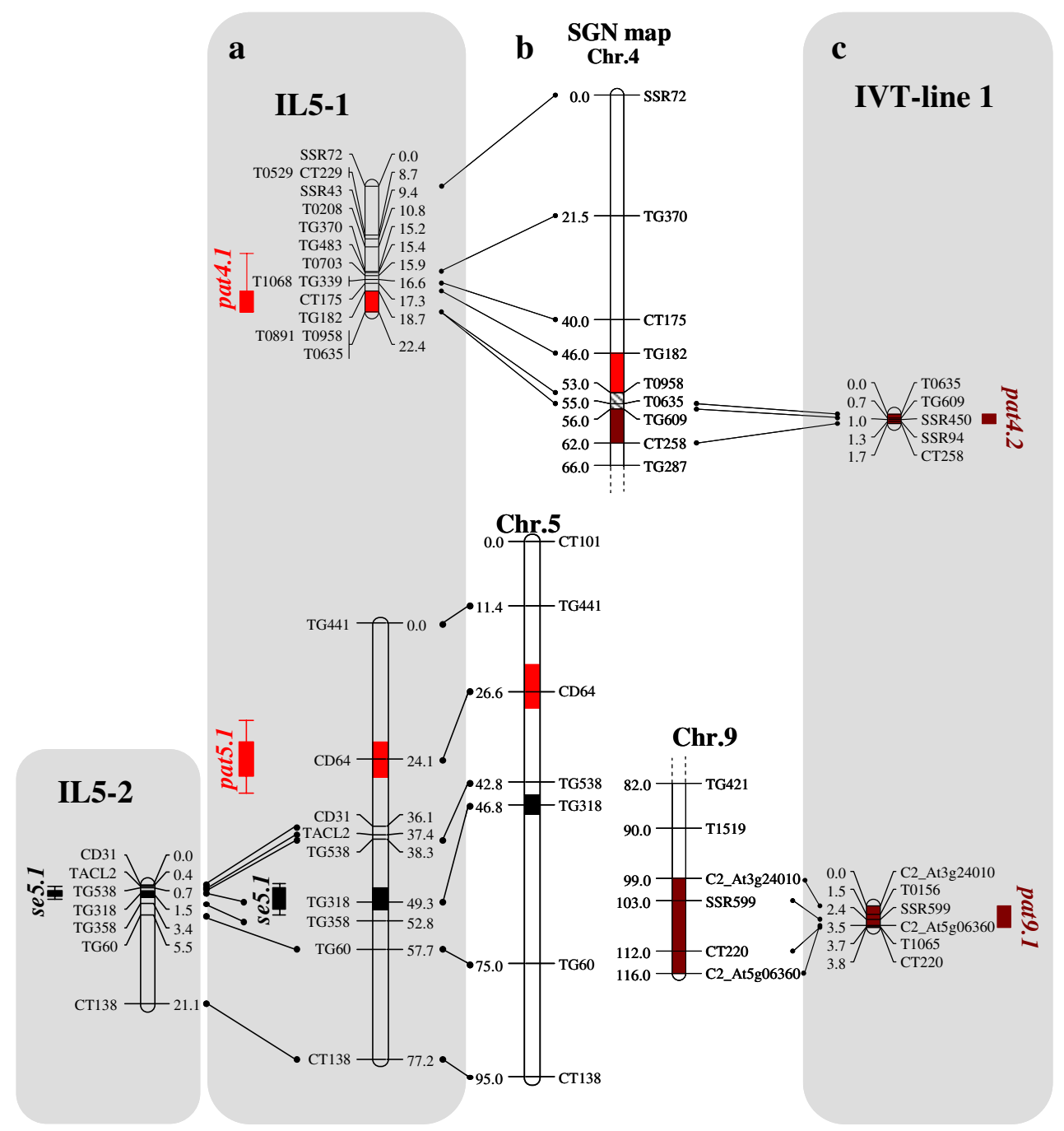

Fig. 3 a Genetic linkage groups of $S H$ introgressions of IL5-1 and IL5-2 developed on population 5-1 and population 5-2. The position of the parthenocarpy QTLs (pat4.1 and pat5.1) and the stigma exsertion locus (se5.1) is indicated on the left of the linkage groups. The se5-1 locus was mapped on population 5-1 and population 5-2. The QTL bars indicate an interval in which the inner, thicker bar, shows a one LOD support confidence interval and the outer bars, thinner, shows a two LOD support confidence interval. b SGN reference map for the short arm and centromeric region of chromosome 4 , the complete chromosome 5 and the telomeric region of the

observations pat4.1 can be considered as the major QTL because it accounts for most of the effect on the parthenocarpy level, and pat5.1 can be considered as a minor QTL that affects the parthenocarpy level of the pat4.1 alleles. Model 1 was chosen among the simple linear models, because in this model the variable is explained by one major QTL, which effect is depending on one minor QTL. The coefficient of correlations for Spring/Summer and Winter were 64.8 and $57.9 \%$, respectively, which confirms that model 1 was appropriate to this case. The details of this model are given in Table 4. long arm of chromosome 9 (http://sgn.cornell.edu). The putative positions of the identified QTLs are represented in it. Because the one LOD confidence interval of pat4.1 and pat4.2 are overlapping, this overlap is indicated with dashed lines. c Genetic linkage groups of chromosome 4 and chromosome $9 \mathrm{SH}$ introgressions of IVT-line 1 developed on the $\mathrm{F}_{2}$ population. The position of the parthenocarpy QTLs (pat4.2 and pat9.1) is indicated on the right of the linkage groups. Map positions are given in cM. Maps and QTL alignments were performed with MapChart

\section{Characterization and mapping of functional sterility}

Functional sterility, procured by stigma exsertion (Fig. 1), was evaluated in populations 5-1 and 5-2 at pre-anthesis on the third cluster. $S H$ flowers have exserted stigmas, whereas the stigma of cv. Moneymaker ( $S L$ ) flowers is inside the anther cone at pre-anthesis. Interval mapping showed one QTL for stigma exsertion, on chromosome 5 introgressions of IL5-1 and IL5-2 (Fig. 3a) linked to marker TG318. We named this locus se5.1 due to its position on chromosome 5 . Plants homozygous for the $\mathrm{SH}$ introgression at marker 
Table 2 Phenotypic analysis of pat4.1 and the stigma exsertion locus, detected by interval mapping in population 5-1 for pat4.1 and populations 5-1 and 5-2 for the stigma exsertion locus

\begin{tabular}{llcccc}
\hline Trait & Genotype & & LOD $^{\mathrm{b}}$ & Explained variation $(\%)$ \\
\cline { 2 - 5 } & $S L / S L\left(n^{\mathrm{c}}\right)$ & $S L / S H(n)$ & $S H / S H(n)$ & & 48.9 \\
\hline Logit (parthenocarpy level) & $-1.73(16)$ & $-0.40(45)$ & $-0.18(13)$ & 10.8 & 39.8 \\
Parthenocarpy level (\%) $^{\mathrm{a}}$ & 1.8 & 28.3 & $2.04(64)$ & 19.23 & 47.9 \\
Stigma exsertion (mm) & $-0.53(3)$ & $0.45(73)$ & & \\
\hline
\end{tabular}

\section{SL, Solanum lycopersicum; SH, Solanum habrochaites}

a The logit numbers are transformed back into parthenocarpy level (percentages)

b The highest LOD score was displayed on markers T0958, T0891 and T0635

c $n$ number of individuals per genotype category

${ }^{d}$ Mapping data calculated by combining populations 5-1 and 5-2

TG318 produced flowers with stigmas significantly more exserted than heterozygous or homozygous $S L$ plants at that marker (Table 2).

In order to study whether the stigma exsertion, observed in IL5-1 and IL5-2, could fully prevent self-pollination, we characterized qualitatively the population 5-2 for the presence or absence of seeded fruits: plants without seeded fruits over the five characterized clusters were differentiated from plants producing at least one fruit with seeds. Population 5-1 was not used for this purpose, because this population was also segregating for parthenocarpy which influences the setting of fruits with seeds. The characterized trait, "presence or absence of seeded fruits", cosegregated with CAPS marker TG318 on chromosome 5 and thus with the QTL for stigma exsertion. This demonstrated that stigma exsertion could fully prevent selfpollination, thus seeded fruits setting.

\section{IVT-line 1}

In a first step towards the mapping of the parthenocarpy trait present in IVT-line 1, we wanted to identify the positions of the $S H$ introgressions. We screened a large number of known $S$. habrochaites AFLP markers on IVTline 1. Seven $S H$ introgressions could clearly be identified, on chromosomes 4, 5, 6, 9 and 11. Only introgressions where known $S$. habrochaites AFLP markers were present

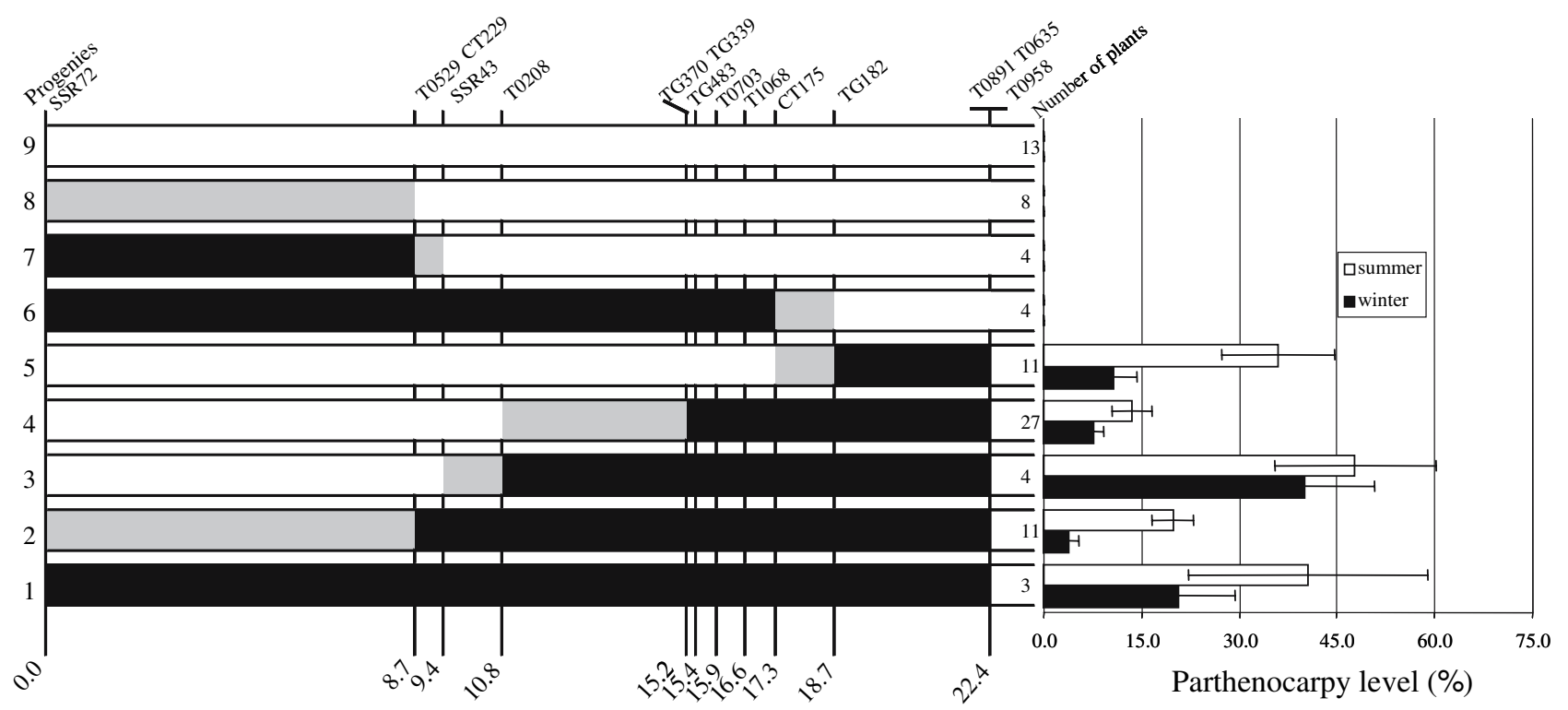

Fig. 4 Left graphical genotypes of $\mathrm{BC}_{5} \mathrm{~S}_{2}$ progenies for the short arm of chromosome 4. "Black" stands for homozygous $\mathrm{SH}$, "white" for homozygous $S L$ and "grey" for unknown because the exact position of the recombination between the two closest flanking markers is unknown. Numbers under the bars indicate the position of the above- mentioned markers, in $\mathrm{cM}$. The reference number of each progeny is indicated at the far left of the graphical genotypes and the number of plants per progeny at the far right. Right average parthenocarpy level (percentages) over the first five clusters per specific progeny, in Spring/Summer (white) and Winter (black), indicated with SE bars 
Table 3 Observed parthenocarpy level (percentages) for each combination of pat4.1 and pat5.1 alleles, in the $\mathrm{BC}_{5} \mathrm{~S}_{2}$, in $\mathrm{Spring}_{\mathrm{S}} \mathrm{Summer}$ and Winter

\begin{tabular}{|c|c|c|c|c|c|c|c|c|c|c|c|c|c|c|c|}
\hline \multirow[t]{3}{*}{ pat4.1 } & \multicolumn{5}{|c|}{ pat5.1 (SL/SL) } & \multicolumn{5}{|c|}{ pat5.1 (SL/SH) } & \multicolumn{5}{|c|}{ pat5.1 $(\mathrm{SH} / \mathrm{SH})$} \\
\hline & \multicolumn{2}{|c|}{ Spring/Summer } & \multicolumn{2}{|c|}{ Winter } & \multirow[t]{2}{*}{$n$} & \multicolumn{2}{|c|}{ Spring/Summer } & \multicolumn{2}{|c|}{ Winter } & \multirow[t]{2}{*}{$n$} & \multicolumn{2}{|c|}{ Spring/Summer } & \multicolumn{2}{|c|}{ Winter } & \multirow[t]{2}{*}{$n$} \\
\hline & Mean & $\mathrm{SE}$ & Mean & SE & & Mean & SE & Mean & SE & & Mean & $\mathrm{SE}$ & Mean & SE & \\
\hline$S L / S L$ & 0.0 & 0.0 & 0.0 & 0.0 & (13) & 0.0 & 0.0 & 0.0 & 0.0 & (6) & 0.0 & 0.0 & 0.0 & 0.0 & (14) \\
\hline$S L / S H$ & 4.1 & 2.3 & 2.4 & 1.2 & (7) & 4.8 & 4.8 & 16.1 & 13 & (2) & 45.5 & 0.0 & 37.5 & 0.0 & (1) \\
\hline SH/SH & 11.6 & 1.9 & 4.6 & 0.9 & (41) & 41.0 & 6.4 & 14.8 & 3.0 & (13) & 46.0 & 6.7 & 27.2 & 6.6 & (9) \\
\hline
\end{tabular}

$S E$ standard error; $n$ number of individuals per genotype; SL, Solanum lycopersicum; SH, Solanum habrochaites

can potentially be retrieved, therefore we cannot exclude the possible presence of other, small $S H$ introgressions.

An $\mathrm{F}_{2}$ population composed of 160 plants, coming from the cross between IVT-line 1 and $S L \mathrm{cv}$. Moneymaker, was grown, and the parthenocarpy level of the plants was evaluated in Spring 2006 in Wageningen, The Netherlands, following the same procedure as previously described. The parthenocarpy level in the $\mathrm{F}_{2}$ population ranged from 0 to $97 \%$. About $44 \%$ of the plants did not produce any parthenocarpic fruit (data not shown). Fruits with and without seeds, within a same cluster were significantly different in size $(P<0.05)$, with averages of 4.36 and $4.11 \mathrm{~cm}$, respectively.

Because of the presence of an $\mathrm{SH}$ introgression around the centromere of chromosome 4 , where pat4.1 was previously mapped, we hypothesized that parthenocarpy in IVT-line 1 might also be under the control of pat4.1 or an allelic variant of it. After confirming that marker T0635 was in the $S H$ introgression of IVT-line 1, we tested the association between marker T0635 (peak marker for pat4.1) and the segregation of parthenocarpy observed in the $F_{2}$ population. This association was highly significant. However, not all plants homozygous $S H$ for T0635 produced parthenocarpic fruits, which may be due to the mode of action of another locus. To localize this other locus, we screened AFLP primer combinations on the set of $F_{2}$ plants homozygous $\mathrm{SH}$ at the T0635 locus. One AFLP marker, P18M51-219 was clearly associated with the parthenocarpy level of the selected plants and was known to be located in

Table 4 Significance of model 1 and estimates of the parameters

\begin{tabular}{lll}
\hline & Spring/Summer & Winter \\
\hline Probability of $\mathrm{F}$ & $<0.001$ & $<0.001$ \\
Coefficient correlation $(\%)$ & 64.8 & 57.9 \\
Constant $(\mu)$ & -6.801 & -6.658 \\
Effect of $S H$ allele of pat4.1 $\left(\alpha_{j}\right)$ & & \\
When pat5.1 is $\operatorname{SL} / S L\left(\alpha_{1}\right)$ & 1.782 & 1.235 \\
When pat5.1 is $\operatorname{SL} / \mathrm{SH}\left(\alpha_{2}\right)$ & 3.101 & 2.439 \\
When pat5.1 is $\operatorname{SH} / S H\left(\alpha_{3}\right)$ & 3.408 & 2.787 \\
\hline
\end{tabular}

a chromosome 9 introgression. Parthenocarpy in IVT-line 1 is therefore under the control of at least two QTLs, one located near the centromere of chromosome 4 and one near the telomere of the long arm of chromosome 9.

To map these two parthenocarpy loci more accurately, we developed a linkage map for the introgressions on chromosomes 4 and 9 (Fig. 3c). The borders of the introgression on chromosome 4 were between markers T0635 and T0958 and between CT258 and TG287. On chromosome 9, the border of the introgression was located between markers T1519 and C2_At3g24010 and likely spanned the rest of the telomeric region of the long arm of chromosome 9. The two linkage groups were generated using eight CAPS markers, converted from RFLP or COS sequences and three SSR markers (SOL Genomics Network database). The order of the markers on chromosome 4 was identical as in the SGN reference map. In the introgression of chromosome 9 , few inversions of marker orders were observed. The introgression of chromosome 4 spanned $1.7 \mathrm{cM}$ ( $7 \mathrm{cM}$ on the SGN reference map) and the introgression on chromosome 9 was $3.8 \mathrm{cM}(17 \mathrm{cM}$ in the reference map). This means that the map distances observed in the introgressions of IVT-line 1 were reduced to $25 \%$ of the distances of the same intervals, presented on the SGN reference map.

Parthenocarpy was mapped using MapQTL. We used a logit scale of the parthenocarpy level to improve the normality of the distribution. Because of the small genetic sizes of the two introgressions on chromosomes 4 and 9, both complete introgressions were highly significant and it was not possible to narrow down the position of the two parthenocarpy QTLs. These two parthenocarpy QTLs are hereafter denoted as pat4.2 (on chromosome 4) and pat9.1 (on chromosome 9). Because it was not possible to narrow down the confidence intervals of pat4.2 and pat9.1, we excluded the plants recombinant for one or both introgressions in the following analysis, and plants with an ambiguous scoring, in order to improve the accuracy of the calculations. In total, out of $160 \mathrm{~F}_{2}$ plants, 137 plants were selected. An ANOVA showed that both QTLs had a highly significant effect on parthenocarpy and also the interaction 
Table 5 Observed and predicted parthenocarpy levels (percentage) for each combination of pat 4.2 and pat9.1 alleles, in the $\mathrm{F}_{2}$ population $(n=137)$

\begin{tabular}{|c|c|c|c|c|c|c|c|c|c|}
\hline \multirow[t]{2}{*}{ pat4.2 } & \multicolumn{3}{|c|}{ pat9.1 (SL/SL) } & \multicolumn{3}{|c|}{ pat9.1 (SL/SH) } & \multicolumn{3}{|c|}{ pat9.1 $(\mathrm{SH} / \mathrm{SH})$} \\
\hline & Pred. $^{\mathrm{a}}$ & Obs. $^{\mathrm{b}}$ & $\mathrm{SE}(n)$ & Pred. & Obs. & $\mathrm{SE}(n)$ & Pred. & Obs. & $\mathrm{SE}(n)$ \\
\hline$S L / S L$ & 0.0 & 0.3 & $0.2(14)$ & 0.0 & 0.8 & $0.4(24)$ & 0.0 & 0.4 & $0.4(4)$ \\
\hline$S L / S H$ & 0.0 & 0.2 & $0.2(14)$ & 0.1 & 12.3 & $2.7(40)$ & 87.5 & 65.2 & $6.0(12)$ \\
\hline $\mathrm{SH} / \mathrm{SH}$ & 0.0 & 0.0 & $0.0(8)$ & 7.1 & 29.2 & $4.4(17)$ & 97.1 & 76.6 & $12.8(4)$ \\
\hline
\end{tabular}

SL, Solanum lycopersicum; SH, Solanum habrochaites

a Predicted effect from model 2 (presented in "Materials and methods"), initially calculated in logit scale, transformed back into percentages

b Observed mean for each combination of alleles (percentages)

between both QTLs was highly significant. Therefore among simple linear models we selected one in which the observed variation is explained by the interaction between two QTLs (model 2). The coefficient of correlation $\left(R^{2}\right)$ was $73.7 \%$, which confirms that the model chosen fitted well the observed variation. The observed and predicted effect, based on model 2, of the different allele's combinations between pat4. 2 and pat 9.1 is presented in Table 5 . The absence of $S H$ allele in either of the pat4.2 or pat9.1 loci results in the absence of parthenocarpic fruit. The highest level of parthenocarpy is obtained when both QTLs are homozygous $S H$ (76\% of parthenocarpy level).

\section{Candidate gene analysis}

One main parthenocarpy gene, known as $f w f$ or $A R F 8$, was recently isolated in Arabidopsis (Goetz et al. 2006). The use of COS markers in the present mapping work and the increasing availability of BAC sequence information linked to tomato markers, allowed us to study the potential microsynteny between the ARF8 Arabidopsis region and the tomato parthenocarpy QTLs identified in this study. Arabidopsis orthologs of markers T0953 and C2_AT5G37360, both located in the pat4.1-pat4.2 region, were found to be closely linked to ARF8 (Table 6). In addition we found one extra Arabidopsis ortholog in the sequence of BAC clone HBa311A10, linked to marker
C2_AT3G54770 in the pat4.1-pat4.2 region, also located near ARF8. Eventually we could draw a microsynteny map that highlights the hypothesis that ARF8 may well be a homolog to pat4.1-pat4.2 (Fig. 5).

\section{Discussion}

We studied parthenocarpy in an IL, named IL5-1, of $S$. habrochaites in a $S$. lycopersicum genetic background. At least two QTLs were responsible for parthenocarpy in this line: one major QTL, referred to as pat4.1, located close to the centromere of chromosome 4 , and one minor QTL, referred to as pat5.1, on the short arm of chromosome 5 . The pat4.1 gene was initially mapped in a $\mathrm{BC}_{5} \mathrm{~S}_{1}$ population, enriched for plants with functional sterility. The pat5.1 locus was later on mapped in another set of specific progenies. The model used to explain the phenotypic variation in the selected progenies improves the understanding of the genetic control of parthenocarpy in this IL. In this model the variation is explained by a major QTL, pat4.1 and a minor QTL, pat5.1. The effect of pat4.1 depends on the number of $S H$ alleles at the pat5.1 locus. When at least one $S H$ allele is present at the pat5.1 locus, the effect of pat4.1 is higher. There is hardly difference in the effect of pat4. 1 between plants carrying one or two $\mathrm{SH}$ alleles at the pat5.1 locus, which means that pat5.1 is a dominant QTL (Table 4). This explains why pat5.1 was not detected in the

Table 6 Arabidopsis orthologs of tomato markers in the pat4.1-pat4.2 region compared to ARF8

\begin{tabular}{|c|c|c|c|c|c|}
\hline \multicolumn{2}{|l|}{ Tomato } & \multicolumn{4}{|c|}{ Arabidopsis genes } \\
\hline Marker Chr.4 & Position $(\mathrm{cM})$ & Accession no. ${ }^{b}$ & Gene name & Chr. & AGI coordinates (bases) \\
\hline T0953 & 49.0 & AT5G37370 & & 5 & $14829915-14833750$ \\
\hline $\mathrm{HBa} 311 \mathrm{~A} 10^{\mathrm{a}}$ & 55.3 & AT5G36905 & & 5 & $14575028-14577414$ \\
\hline \multirow[t]{2}{*}{ C2_AT5G37360 } & 56.0 & AT5G37360 & & 5 & $14822459-14825466$ \\
\hline & & AT5G37020 & $A R F 8$ & 5 & $14647258-14651617$ \\
\hline
\end{tabular}

${ }^{a}$ BAC clone HBa311A10 is linked to marker C2_AT3G54770 which has been mapped at $55.3 \mathrm{cM}$ on Chr. 4 on the EXPEN2000 reference map

${ }^{\mathrm{b}}$ Arabidopsis orthologs were found in the SOL genomics network (http://www.sgn.cornell.edu) 
Fig. 5 Microsynteny between the pat4.1-pat4.2 tomato region and ARF8 Arabidopsis region. A zoom in the two regions of interest, with the microsyntenic relations, is highlighted in grey

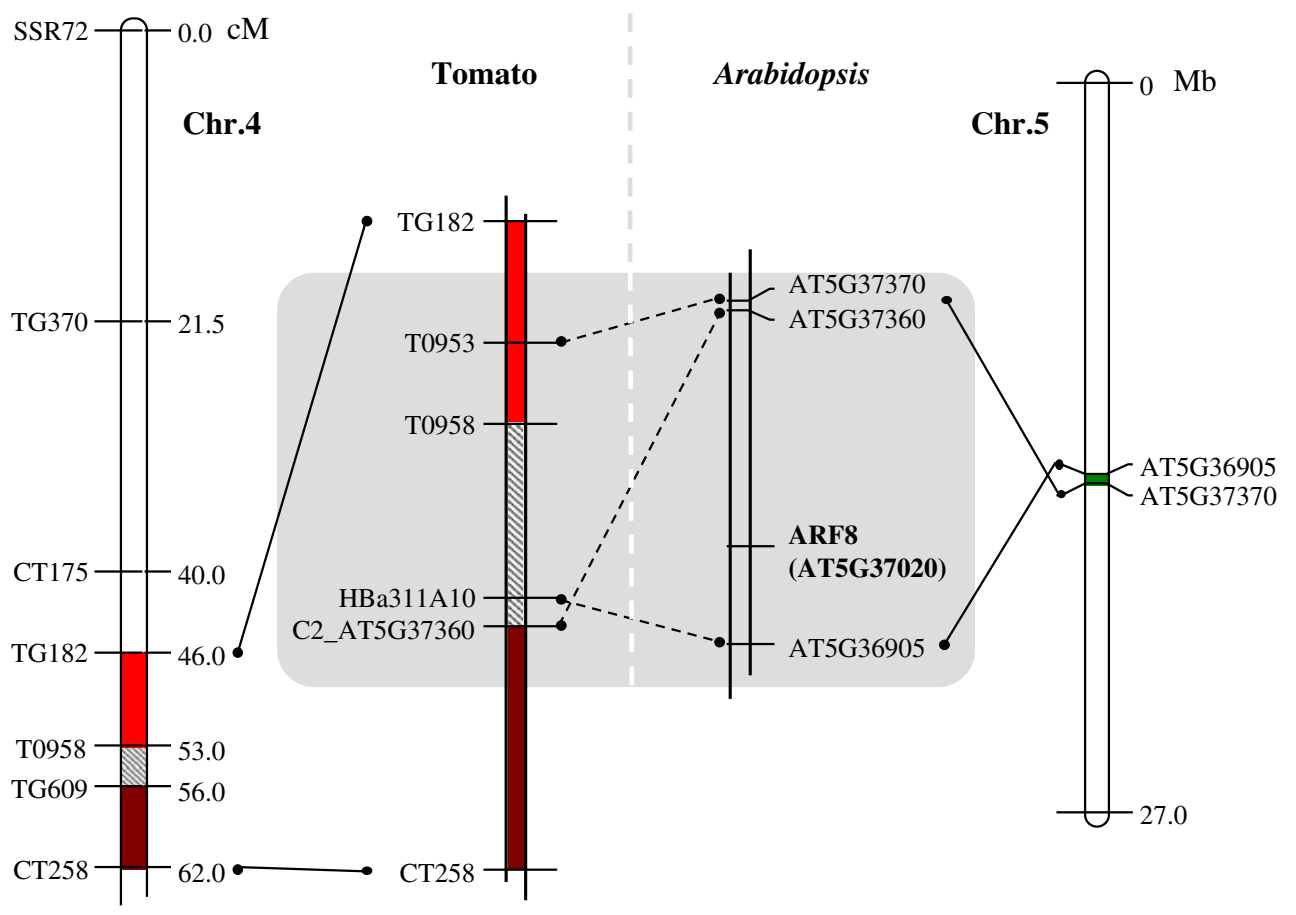

$\mathrm{BC}_{5} \mathrm{~S}_{1}$ population, because the enrichment for functional sterility also resulted in an enrichment of $S H$ alleles at the pat5.1 locus.

We mapped the stigma exsertion locus close to marker TG318, on chromosome 5. When the presence of seeded fruits was considered as a qualitative trait, it co-segregated with marker TG318. This supports the hypothesis that the exserted stigma prevents self-pollination and therefore prevents seeded fruits setting. Bernacchi and Tanksley (1997) characterized stigma exsertion in an IL of S. habrochaites acc. LA1777 in a S. lycopersicum genetic background. They mapped the main stigma exsertion locus on chromosome 2 and did not report a functional sterility locus on chromosome 5. However, the ILs of Monforte and Tanksley (2000) do not cover the complete genome and amongst others the central part of chromosome 5 is missing. It is therefore impossible at this stage to speculate whether the stigma exsertion locus identified in our study is specific to accession LYC4, or inherent to the S. habrochaites species.

The position of pat4.1 led to the identification of pat4.2, one of the two parthenocarpy QTLs identified in IVT-line 1. Eventually, we identified two QTLs, one close to the centromere of chromosome 4, referred to as pat4.2, and one close to the telomere of the long arm of chromosome 9, referred to as pat9.1. Both QTLs are of equal importance in the parthenocarpic expression. It is not clear at this stage whether pat4.1 and pat4.2 refer to the same gene. Some minor differences can be observed between both genetic controls of parthenocarpy, but the allelic possibility between pat4.1 and pat4.2 cannot be discarded: The presence of pat4.1 alone is enough to obtain a certain level of parthenocarpy, but pat4.2 alone does not give any parthenocarpic fruit development. In addition we observed that the size of seedless fruits produced in pat4.1/pat5.1 plants was similar to the size of the fruits with seeds on the same material, but a significant smaller size was observed in the pat4.2/pat9.1 material. To give a definitive answer to the question whether pat4.1 and pat4.2 are allelic, we need to fine-map both QTLs. However, fine mapping will be hampered by the strong suppression of recombination, especially in the small introgressed fragment of IVT-line 1, giving rise to the reductions of map distances observed in this study, and by the centromeric position of these parthenocarpy QTLs. Reduction in recombination has been observed previously in introgressed intervals from wild tomato species (Chetelat and Meglic 2000; Monforte and Tanksley 2000; Rick 1969). Sequence divergence between wild and cultivated Solanum species has been suggested as cause for this phenomenon (Paterson et al. 1990).

The increasing availability of sequence information provided by the sequencing of BAC clones anchored to molecular markers, and the recent development and mapping of COS markers helped us to study the syntenic relationship between tomato and Arabidopsis at parthenocarpy QTLs regions. We focused on the potential synteny of the parthenocarpy QTLs regions with the Arabidopsis parthenocarpy locus, $A R F 8$, region. Two COS markers and 
one putative gene of the pat4.1-pat4.2 region were orthologous to Arabidopsis genes closely linked to ARF8 (Fig. 5). This finding provides evidence that pat4.1-pat4.2 may be homologs of ARF8. However, T0953 and C2_AT5G37360 (both extreme borders of the syntenic map presented in Fig. 5) are distant of $7 \mathrm{cM}$ in the EXPEN2000 reference map, which is, to our knowledge, much larger than in any reported synteny between tomato and Arabidopsis. ARF8 has been found to act as an inhibitor for further carpel development, in Arabidopsis, in the absence of fertilization (Goetz et al. 2006). A lesion in ARF8, found in the fruit without fertilization mutant ( $f w f$ ) has resulted in the uncoupling of fruit development from pollination and fertilization and therefore has given rise to seedless fruit. Further fine mapping and isolation of the parthenocarpy QTLs, identified in this study, are necessary to confirm the potential homology with $A R F 8$. Recently the tomato ARF8 gene has been isolated (Goetz et al. 2007). Expression of an aberrant form of Arabidopsis ARF8, in tomato conferred parthenocarpy, which proves that ARF8 is also involved in the control of fruit set in tomato. Sequencing of the ARF8 gene on IL5-1 and IVT-line 1 may already give information on the functionality of this candidate gene in both parthenocarpic lines. In addition, the mapping of the tomato ARF 8 homolog would also be sufficient to discard the possibility of homology between the pat4.1-pat4.2 and $A R F 8$, in case the tomato ARF8 homolog would not cosegregate with the parthenocarpy QTLs.

In the present experiment, the parthenocarpy level was higher with pat4.2/pat9.1 than with pat4.1/pat5.1. With pat4.2/pat9.1, we observed an average of $76.6 \%$ parthenocarpic fruit set in Spring compared to $46 \%$ for pat4.1/ pat5.1, in Summer. In both cases, the higher the number of parthenocarpy alleles is, the higher the level of parthenocarpy. We can only speculate about the level of parthenocarpy in one single line with all four parthenocarpy QTLs. However, the development of such a line is now possible by molecular assisted selection, using the above-mentioned markers. A too strong parthenocarpic expression often results in a fruit set that precedes anthesis as observed in the pat mutant Soressi (Mazzucato et al. 1998). Therefore in such material the production of seeds, when needed, is hampered even when pollination is promoted. This limits the interest for breeders who ultimately want to commercialize seeds. It would be valuable to study the level of parthenocarpy in a hybrid that combines several parthenocarpy QTLs, most or all of them in heterozygous state. If such a hybrid displays a high parthenocarpy level, it would be possible to commercialize it by developing two parental lines that carry different parthenocarpy QTLs, or one parental line, used as male parent, that combines most of the parthenocarpy QTLs in homozygous and the other parental line, used as female parent, carrying only few parthenocarpy QTLs, thus with a high level of seed production. Of course a reasonable level of seed production in the male parent is also needed. A detailed study of the parthenocarpy level of these new sources of parthenocarpy in different environments, different genetic background, as well as combining the four parthenocarpy QTLs or making new combinations will allow comparisons with the reference parthenocarpy lines such as Soressi (pat), Severianin (pat-2) and RP75/59 (pat3/pat-4). This will show the potential of these newly identified QTLs for practical breeding.

So far, only one parthenocarpy gene, pat, was mapped in tomato (Beraldi et al. 2004). In this study we mapped four parthenocarpy QTLs in tomato, which provides valuable information for the development of parthenocarpic tomato varieties. The isolation of the main QTLs will greatly enhance our understanding of the control of fruit set in tomato and offer possibilities, considering the high level of similarities within the Solanaceae, to develop seedless fruits in other economically important species.

Acknowledgments This project was sponsored by Western Seed BV, The Netherlands.

Open Access This article is distributed under the terms of the Creative Commons Attribution Noncommercial License which permits any noncommercial use, distribution, and reproduction in any medium, provided the original author(s) and source are credited.

\section{References}

Beraldi D, Picarella ME, Soressi GP, Mazzucato A (2004) Fine mapping of the parthenocarpic fruit (pat) mutation in tomato. Theor Appl Genet 108:209-216

Bernacchi D, Tanksley SD (1997) An interspecific backcross of Lycopersicon esculentum $\times$ L. hirsutum: linkage analysis and a QTL study of sexual compatibility factors and floral traits. Genetics 147:861-877

Brouwer DJ, St. Clair DA (2004) Fine mapping of three quantitative trait loci for late blight resistance in tomato using near isogenic lines (NILs) and sub-NILs. Theor Appl Genet 108:628-638

Chen K-Y, Tanksley SD (2004) High-resolution mapping and functional analysis of se2.1: a major stigma exsertion quantitative trait locus associated with the evolution from allogamy to autogamy in the genus Lycopersicon. Genetics 168:15631573

Chetelat RT, Meglic V (2000) Molecular mapping of chromosome segments introgressed from Solanum lycopersicoides into cultivated tomato (L. esculentum). Theor Appl Genet 100:232-241

Coaker GL, Francis DM (2004) Mapping, genetic effects, and epistatic interaction of two bacterial canker resistance QTLs from Lycopersicon hirsutum. Theor Appl Genet 108:1047-1055

Finkers R, van den Berg P, van Berloo R, ten Have A, van Heusden AW, van Kan JA, Lindhout P (2007a) Three QTLs for Botrytis cinera resistance in tomato. Theor Appl Genet 114:585-593

Finkers R, van Heusden AW, Meijer-Dekens F, van Kan JA, Maris P, Lindhout P (2007b) The construction of a Solanum habrochaites LYC4 introgression line population and the identification of 
QTLs for resistance to Botrytis cinera. Theor Appl Genet 114:1071-1080

Fulton TM, van der Hoeven R, Eannetta NT, Tanksley SD (2002) Identification, analysis, and utilization of conserved ortholog set markers for comparative genomics in higher plants. Plant Cell 14:1457-1467

George W, Scott J, Spilttstoesser W (1984) Parthenocarpy in tomato. Hortic Rev 6:65-84

Goetz M, Vivian-Smith A, Johnson SD, Koltunow AM (2006) AUXIN RESPONSE FACTOR8 is a negative regulator of fruit initiation in Arabidopsis. Plant Cell 18:1873-1886

Goetz M, Hooper LC, Johnson SD, Rodrigues JC, Vivian-Smith A, Koltunow AM (2007) Expression of aberrant forms of AUXIN RESPONSE FACTOR8 stimulates parthenocarpy in Arabidopsis and tomato. Plant Physiol 145:351-366

Gorguet B, van Heusden AW, Lindhout P (2005) Parthenocarpic fruit development in tomato. Plant Biol 7:131-139

Gorguet B, Schipper D, van Heusden AW, Lindhout P (2006) High resolution fine mapping of $p s-2$, a mutated gene conferring functional male sterility in tomato due to non-dehiscent anthers. Theor Appl Genet 113:1437-1448

Mazzucato A, Taddei AR, Soressi GP (1998) The parthenocarpic fruit (pat) mutant of tomato (Lycopersicon esculentum Mill.) sets seedless fruits and has aberrant anther and ovule development. Development 125:107-114

Monforte AJ, Tanksley SD (2000) Development of a set of near isogenic and backcross recombinant inbred lines containing most of the Lycopersicon hirsutum genome in a L. esculentum genetic background: a tool for gene mapping and gene discovery. Genome 43:803-813

Mueller LA, Solow TH, Taylor N, Skwarecki B, Buels R, Binns J, Lin C, Wright MH, Ahrens R, Wang Y, Herbst EV, Keyder ER, Menda N, Zamir D, Tanksley SD (2005) The SOL genomics network. A comparative resource for Solanaceae biology and beyond. Plant Physiol 138:1310-1317
Myburg AA, Remington DL (2000) Protocol for high-throughput AFLP analysis using LI-COR IR2 automated sequencer. NC State Forest Biotech AFLP protocol-Sept 2000

Paterson AH, De-Verna JW, Lanini B, Tanksley SD (1990) Fine mapping of quantitative trait loci using selected overlapping recombinant chromosomes, in an interspecific cross of tomato. Genetics 124:735-742

Picken AJF (1984) A review of pollination and fruit set in the tomato (Lycopersicon esculentum Mill.). J Hortic Sci 59:1-13

Rick CM (1969) Controlled introgression of chromosomes from Solanum pennellii into Lycopersicon esculentum: segregation and recombination. Genetics 62:753-768

Steward CN, Via LE (1993) A rapid CTAB DNA isolation technique useful for RAPD fingerprinting and other PCR applications. Biotechniques 14:748-750

Tanksley SD, Ganal MW, Prince JP, de Vicente MC, Bonierbale MW, Broun P, Fulton TM, Giovannoni JJ, Grandillo S, Martin GB, Messeguer R, Miller JC, Miller L, Paterson AH, Pineda O, Roder MS, Wing RA, Wu W, Young ND (1992) High density molecular linkage maps of tomato and potato genomes. Genetics 132:1141-1160

Van Ooijen JW (1999) LOD significance thresholds for QTL analysis in experimental populations of diploid species. Heredity 83:613624

Van Ooijen JW (2004) MapQTL® 5, software for the mapping of quantitative trait loci in experimental populations. Kyazma B.V., Wageningen

Van Ooijen JW, Voorrips RE (2001) JoinMap® 3.0, software for the calculation of genetic linkage maps. Plant Research International, Wageningen

Wang H, Qi M, Cutler AJ (1993) A simple method of preparing plant samples for PCR. Nucleic Acids Res 21:4153-4154

Zijlstra S (1985) Parthenocarpie in tomaat; twee nieuwe lijnen uit soortkruising. Zaadbelangen 4:92-94 